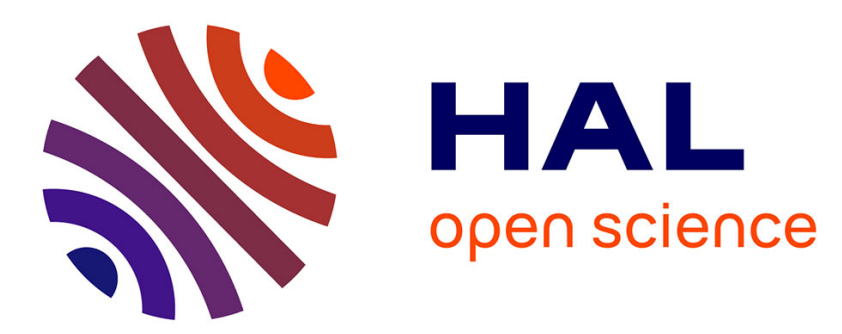

\title{
Modèle aéroélastique appliqué à la déformation d'un gréement
}

\author{
Frédéric Hauville, Mathieu Durand, Yann Roux
}

\section{To cite this version:}

Frédéric Hauville, Mathieu Durand, Yann Roux. Modèle aéroélastique appliqué à la déformation d'un gréement. European Journal of Environmental and Civil Engineering, 2008, 12 (5), pp.549-560. hal-02140722

\section{HAL Id: hal-02140722 \\ https://hal.science/hal-02140722}

Submitted on 27 May 2019

HAL is a multi-disciplinary open access archive for the deposit and dissemination of scientific research documents, whether they are published or not. The documents may come from teaching and research institutions in France or abroad, or from public or private research centers.
L'archive ouverte pluridisciplinaire HAL, est destinée au dépôt et à la diffusion de documents scientifiques de niveau recherche, publiés ou non, émanant des établissements d'enseignement et de recherche français ou étrangers, des laboratoires publics ou privés. 


\title{
Modèle aéroélastique appliqué à la déformation d'un gréement
}

\author{
Frédéric Hauville* ${ }^{*}$ Mathieu Durand ${ }^{* *}$ - Yann Roux ${ }^{* *}$ \\ * Institut de Recherche de l'École Navale - EA3634 \\ B.P. 600, F-29240 Brest Armées, FRANCE \\ frederic.hauville@ecole-navale.fr \\ ** Société K-epsilon, \\ 30 rue Stanislas Girardin, F-76000 Rouen, FRANCE \\ \{mathieu,yann\}@k-epsilon.com
}

RÉSUMÉ. Ce travail est une contribution au développement d'un modèle d'aéroélasticité nonlinéaire, destiné à la prédiction de comportement de surfaces souples de faible épaisseur, soumises à un écoulement de fluide parfait. Ce modèle d'interaction fluide-structure intègre un calcul de structure basé sur un modèle de membrane, à un calcul fluide tridimensionnel instationnaire, basé sur une méthode de singularités associée à une méthode particulaire. Les résultats sur un gréement de J80 sont comparés à des essais in situ.

ABSTRACT. This study adds to the development of a non linear aeroelastic model which is destined to predict the behaviour of a thin flexible surface in contact with a flow under the hypothesis of an inviscid fluid. This fluid structure interaction model integrates a structure calculation, based on a membrane model, with a fluid calculation, based on a singularity method associated with a vortex method. The results on a J80 rig are compared to the experiments in situ.

MOTS-CLÉS : Aéroélasticité non-linéaire, membrane, fluide parfait, couplage fluide-structure, expérimentation

KEYWORDS: Non-linea aeroelasticity, membrane, inviscid fluid, fluid-structure interaction, testing 


\section{Introduction}

La recherche de performance, dans la conception et l'optimisation des voiliers de compétition participant à des courses de plus en plus médiatisées, nécessite des études relatives à la modélisation de la mécanique inhérente à la dynamique du voilier (Roux et al., 2005). Ce dernier est une structure complexe à l'interface de deux fluides dont les problèmes hydrodynamique, aérodynamique et structurel sont fortement interdépendants et ont une physique fortement non linéaire. Les travaux réalisés à l'IRENav depuis plusieurs années concernent la modélisation numérique du problème aéroélastique par le couplage entre un code de calcul d'écoulement fluide parfait tridimensionnel instationnaire, basé sur une méthode de singularités associée à une méthode particulaire, et un code de déformation de structure, basé sur une méthode originale de réseaux de fils particulièrement bien adaptée aux tissus orthotropes (Le Maître et al., 1998). Le formalisme de ce modèle filaire est modifié pour prendre en compte la rigidité des surfaces souples dans le biais (Mounoury et al., 2005; Mounoury, 2006). Cette méthode est adaptée au calcul de la déformation des voiles à coupe orientées et les informations nécessaires à sa mise en œuvre (modules élastiques...) sont facilement accessibles. Cependant, l'utilisation croissante de matériaux de plus en plus sophistiqués dans l'élaboration des voiles «moulées » (3DL, D4...) a nécessité le développement d'un autre modèle de déformation de structure qui repose sur la théorie de la membrane avec des éléments à trois nouds de type CST (Imbert, 1995). Dans un cadre plus général, ces développements s'inscrivent dans la problématique de compréhension des mécanismes physiques intervenant dans la réponse dynamique de systèmes de fluide en écoulement autour de structures solides déformables. Les équations des modèles fluide et solide sont présentées dans une première partie. La deuxième partie est consacrée à la procédure de couplage permettant d'assurer un avancement en temps des solutions dans les deux domaines. La dernière partie évoque les premiers travaux expérimentaux in situ sur un gréement, dans l'optique, à terme, de valider des modèles couplés fluide-structure proposés par une confrontation des résultats d'une simulation numérique aux mesures.

\section{Les modèles}

\subsection{Modèle fluide}

Le calcul de l'écoulement autour des voiles est effectué dans l'hypothèse d'un fluide parfait incompressible, par la méthode particulaire développé par Rehbach (Rehbach, 1979 - 1980; Charvet et al., 1996). La modélisation de l'écoulement permet de calculer la vitesse du fluide en tout point et d'en déduire la force aérodynamique exercée sur la surface portante par le fluide. La résolution autour de la surface portante est traitée par une méthode des singularités (Hauville et al., 2003). Celle-ci requiert de ne mailler que la surface de l'obstacle et non l'ensemble du domaine de l'écoulement. Cependant, le traitement du sillage peut rapidement devenir coûteux, car l'évaluation d'une part, de la vitesse et la déformation de chaque particule et d'autre part, de l'in- 
tensité des particules émises au bord de fuite à chaque pas de temps peut engendrer un nombre de points de calcul très important. Afin de réduire ce temps de calcul, nous avons mis en œuvre une technique de coalescence des particules décrivant le sillage.

\subsection{Modèle de structure membranaire}

Dans cette partie nous présentons le modèle de structure membranaire permettant de prédire la configuration d'équilibre d'une structure souple de faible épaisseur soumise à un chargement extérieur. Cela revient à déterminer les déformations de la structure, calculées à partir du champ de déplacement, qui conduisent à un ensemble de forces internes capables d'équilibrer les efforts extérieurs. Les grandeurs continues s'expriment en fonction de leurs valeurs aux nœuds à l'aide de fonctions d'interpolation qui dépendent du type d'éléments finis. Notre choix s'est porté sur l'élément CST (Constant Strain Triangular) à trois nœuds (9 degrés de liberté en 3D) sur lequel l'interpolation est linéaire. Cet élément impose également des déformations et des contraintes constantes à l'intérieur de ses frontières. Les tenseurs des déformations et des contraintes ainsi que les forces internes sont calculés dans des repères locaux liés aux éléments. A l'aide de changements de repère et par assemblage des sous-systèmes matriciels, le système d'équation différentielle du problème complet s'écrit sous la forme d'un système matriciel exprimé et résolu dans le repère global.

\subsubsection{Repère global et local}

La structure étant de faible épaisseur et idéalement flexible, nous nous plaçons dans l'hypothèse des contraintes planes $\left(\underline{\sigma}_{3}=\underline{\mathbf{0}}\right)$. Pour chaque élément, un repère local $\left(O^{\prime}, \underline{\boldsymbol{e}}_{x}^{\prime}, \underline{\boldsymbol{e}}_{y}^{\prime}\right)$ noté $\mathcal{R}^{\prime}$ dans lequel les déformations et les forces internes de l'élément sont calculées lui est lié. La structure complète est, quant à elle, étudiée dans un repère global $\left(O, \underline{\boldsymbol{e}}_{x}, \underline{\boldsymbol{e}}_{y}, \underline{\boldsymbol{e}}_{z}\right)$ noté $\mathcal{R}$.

La matrice $\left[\lambda_{i}\right]$ est la matrice de passage du repère local de l'élément $i$ vers le repère global :

$$
\left[\lambda_{i}\right]=\left[\begin{array}{ccc}
i_{x}^{\prime} & j_{x}^{\prime} & k_{x}^{\prime} \\
i_{y}^{\prime} & j_{y}^{\prime} & k_{y}^{\prime}
\end{array}\right]
$$

avec $\left(i_{x}^{\prime}, j_{x}^{\prime}, k_{x}^{\prime}\right)$ et $\left(i_{y}^{\prime}, j_{y}^{\prime}, k_{y}^{\prime}\right)$ les coordonnées des vecteurs unitaires $\underline{\boldsymbol{e}}_{x}^{\prime}$ et $\underline{\boldsymbol{e}}_{y}^{\prime}$ dans le repère global.

La matrice de passage $\left[\Lambda_{i}\right]$, qui permet de transformer les vecteurs généralisés de la structure exprimés dans le repère global en vecteurs généralisés de l'élément $i$ exprimés dans le repère local, est construite à partir de la matrice $\left[\lambda_{i}\right]$. Soit $N_{p}$ le nombre de nœuds, $\left[\Lambda_{i}\right]$ est de dimension $\left(6 \times 3 * N_{p}\right)$, et $\left[\lambda_{i}\right]$ de dimension $(2 \times 3)$.

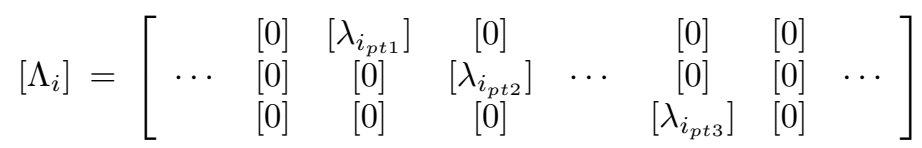




\subsubsection{Champs de déplacements}

On considère un élément triangulaire CST comportant un nœud en chacun de ses sommets, indicé $i=1,2,3$. Tous les points de la surface triangulaire sont repérés dans le plan $\left(O^{\prime}, \underline{\boldsymbol{e}}_{x}^{\prime}, \underline{\boldsymbol{e}}_{y}^{\prime}\right)$ par leur vecteur position courante $\underline{\mathbf{r}}^{\prime}\left(x^{\prime}, y^{\prime}\right)$ tel que :

$$
\underline{\mathbf{r}}^{\prime}\left(x^{\prime}, y^{\prime}\right)=\underline{\boldsymbol{u}}^{\prime}\left(x^{\prime}, y^{\prime}\right)+\underline{\mathbf{r}}_{0}^{\prime}\left(x^{\prime}, y^{\prime}\right)
$$

où $\underline{\mathbf{r}}_{0}^{\prime}\left(x^{\prime}, y^{\prime}\right)$ représente le vecteur position initial du point de coordonnées $\left(x^{\prime}, y^{\prime}\right)$ et $\underline{\boldsymbol{u}}^{\prime}\left(x^{\prime}, y^{\prime}\right)$ son déplacement. Soit, $\left\{q^{\prime}\right\}_{e}$ le vecteur des déplacements généralisés qui regroupe les composantes des déplacements aux nœuds exprimées dans le repère local :

$$
\left\{q^{\prime}\right\}_{e}=\left\{u_{x_{1}^{\prime}}^{\prime} u_{y_{1}^{\prime}}^{\prime} u_{x_{2}^{\prime}}^{\prime} u_{y_{2}^{\prime}}^{\prime} u_{x_{3}^{\prime}}^{\prime} u_{y_{3}^{\prime}}^{\prime}\right\}
$$

Le vecteur des déplacements $\left\{u^{\prime}\right\}_{e}$ peut être exprimé en fonction du vecteur des déplacements généralisés $\left\{q^{\prime}\right\}_{e}$,

$$
\left\{u^{\prime}\right\}_{e}=\left[N^{\prime}\right]_{e}\left\{q^{\prime}\right\}_{e}
$$

où $\left[N^{\prime}\right]_{e}$ est la matrice d'interpolation des déplacements sur les déplacements nodaux.

\subsubsection{Déformations et contraintes}

Les déformations peuvent maintenant être calculées à travers cette expression :

$$
\left\{\epsilon^{\prime}\right\}_{e}=\left[D^{\prime}\right]_{e}\left\{u^{\prime}\right\}_{e}
$$

où $\left[D^{\prime}\right]_{e}$ représente la matrice des opérateurs différentiels permettant de calculer les déformations en fonction des déplacements. Le vecteur des déplacements généralisés $\left\{u^{\prime}\right\}_{e}$, des nœuds de l'élément $e$, est remplacé par l'expression de son champ approché :

$$
\left\{\epsilon^{\prime}\right\}_{e}=\left[D^{\prime}\right]_{e}\left[N^{\prime}\right]_{e}\left\{q^{\prime}\right\}_{e}=\left[B^{\prime}\right]_{e}\left\{q^{\prime}\right\}_{e}
$$

$\left[B^{\prime}\right]_{e}$ est appelé matrice d'interpolation des déformations. On en déduit les contraintes $\left\{\sigma^{\prime}\right\}_{e}$ en fonction des déplacements aux nœuds :

$$
\left\{\sigma^{\prime}\right\}_{e}=\left[C^{\prime}\right]_{e}\left\{\epsilon^{\prime}\right\}_{e}=\left[C^{\prime}\right]_{e}\left[B^{\prime}\right]_{e}\left\{q^{\prime}\right\}_{e}
$$

$\left[C^{\prime}\right]_{e}$ représente la matrice de comportement de la structure. Ces déformations et contraintes sont exprimées dans le repère local. Elles sont uniformes sur l'ensemble de l'élément. La matrice de rigidité a pour expression :

$$
\left[K^{\prime}\right]_{e}=h A_{e}\left[B^{\prime}\right]_{e}^{T}\left[C^{\prime}\right]_{e}\left[B^{\prime}\right]_{e}
$$

avec $h$ et $A_{e}$, l'épaisseur et l'aire de l'élément. A ce stade, nous pouvons écrire les forces internes de l'élément dans le repère local :

$$
\left\{F^{\prime}\right\}_{e}=\left[K^{\prime}\right]_{e}\left\{q^{\prime}\right\}_{e}
$$


avec :

$$
\left\{q^{\prime}\right\}_{e}=\left\{X^{\prime}\right\}-\left\{X_{\text {initial }}^{\prime}\right\}
$$

La matrice de rigidité joue une rôle important. Elle permet d'exprimer directement l'énergie de déformation et les forces internes en fonction des déplacements.

\subsubsection{La matrice de comportement}

Les tissus modélisés sont un assemblage de familles de fils maintenus par des films isotropes. La matrice de comportement $\left[C^{\prime}\right]_{e}$ du tissu se construit en additionnant les matrices de comportement élémentaires associées à chacune des " sous-structures » composant le tissu. Nous utilisons ici la matrice $\left[C^{*}\right]_{e}=h\left[C^{\prime}\right]_{e}$ plus adaptée aux calculs de voiles. En pratique, les déformations des voiles restent inférieures à $1 \%$. On utilise donc une matrice de comportement identique pendant tout le calcul : ce qui suppose que les angles entre les fibres ne changent pas et que tous les matériaux restent dans leur domaine linéaire.

\subsubsection{Résolution du système}

Le système matriciel exprimant l'équation d'équilibre d'une structure soumise à un ensemble de forces nodales extérieures s'obtient en appliquant le $1^{\text {er }}$ théorème de Castigliano sur l'énergie de déformation :

$$
\left\{F_{\text {ext }}\right\}=[K]\{q\}=\sum_{i=1}^{N_{e}}\left[\Lambda_{i}\right]^{T}\left[K^{\prime}\right]_{i}\left[\Lambda_{i}\right]\{q\}
$$

$\left\{F_{\text {ext }}\right\}$ est ici le vecteur forces extérieures généralisées, $\{q\}$ le vecteur des déplacements généralisés et $[K]$ la matrice de rigidité de la structure complète obtenue en assemblant les matrices de rigidité $\left[K^{\prime}\right]_{i}$ de chaque élément composant la structure. La résolution de ce système permet de déterminer $\{q\}$. Dans le cadre de problèmes non linéaires, la résolution de l'équation d'équilibre précédente n'est pas immédiate. En effet $[K]$ dépend du vecteur des déplacements généralisés $\{q\}$. Le système est résolu par une méthode itérative décrite par l'organigramme figure 1. Le vecteur résidu $\{$ Residu\} correspond aux efforts résiduels aux nœuds de la structure. Il tend vers 0 lorsque l'on tend vers l'équilibre. Le coefficient de relaxation $\zeta$ permet de modérer l'ensemble des déplacements apportés à la structure. Le vecteur des déplacements généralisés $\{q\}^{k}$ représente le déplacement des nœuds de la structure entre les itérations $k-1$ et $k$ et non le déplacement des nœuds entre la configuration courante $\{X\}^{k}$ et la configuration initiale $\left\{X_{\text {initial }}\right\}$. Il est calculé en fonction des efforts résiduels. Le calcul des forces internes se fait donc à travers la relation,

$$
\left\{F_{\text {int }}^{\prime}\right\}_{e}^{k}=\left[K^{\prime}\right]_{e}\left(\{X\}^{k}-\left\{X_{\text {initial }}\right\}\right)
$$

Pour l'étude dynamique, les termes d'accélération sont discrétisés par un schéma de Newmark en introduisant un intervalle $\delta t$ entre deux temps discrets. 


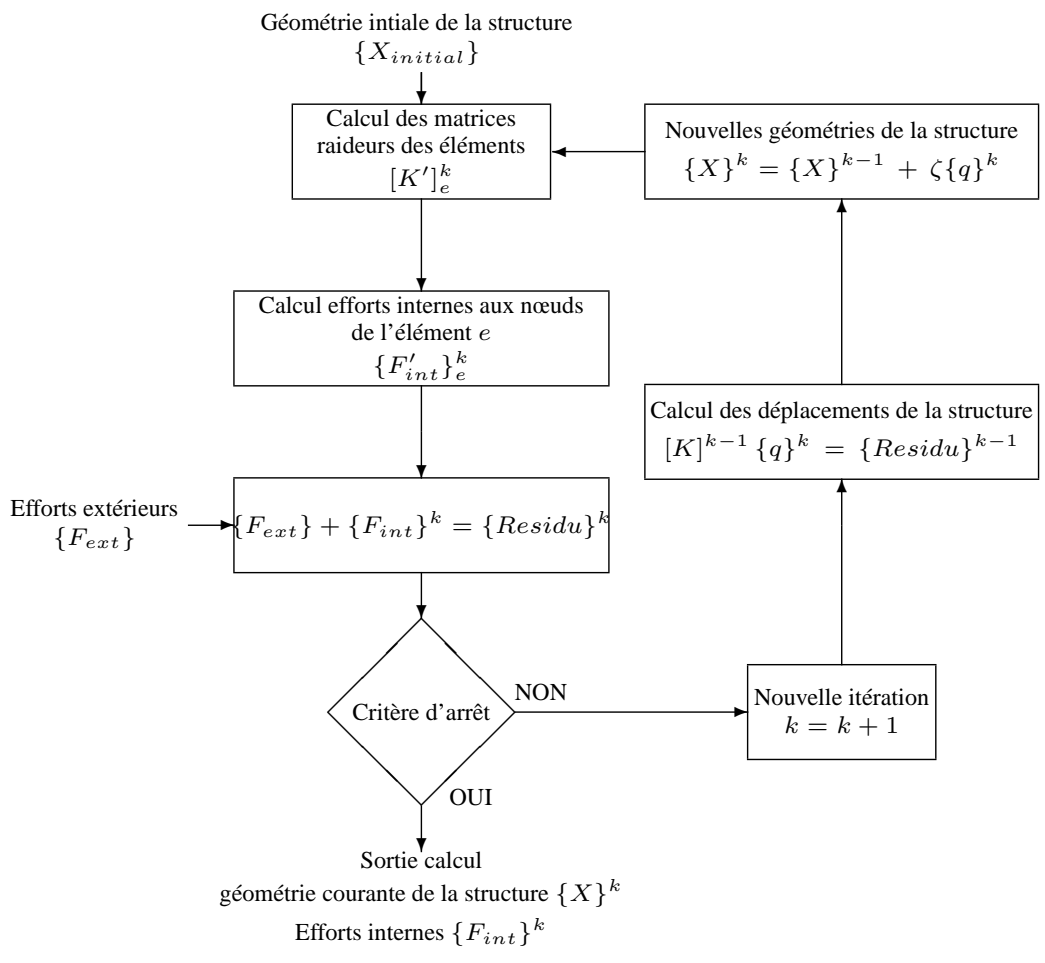

Figure 1. Boucle itérative pour l'équilibre statique

\subsubsection{Les modèles de plis}

Les plis sont pris en compte d'une part, en minimisant l'énergie élastique (dépend du maillage) et d'autre part, en annulant les contraintes de compression dans certaines zones. En effet, lors d'une forte traction sur un tissu, des plis de compressions apparaissent. Ces plis qui naissent de l'instabilité interne entre les efforts de compression et de flexion, sont assimilables à un flambement. Dans l'hypothèse d'un matériau sans rigidité de flexion, le tissu ne supporte aucune compression : il se plisse. Les plis étant de longueur d'ondes infiniment petite, nous utilisons un modèle simple pour simuler ces plis et donc le comportement unilatéral du tissu. Pour vérifier l'état de compression du tissu, nous utilisons la matrice du tenseur des contraintes $\left[\sigma^{*}\right]_{2 \times 2}$ à partir de :

$$
\left\{\sigma^{*}\right\}_{e}=\left[C^{*}\right]_{e}\left\{\epsilon^{\prime}\right\}_{e}
$$

L'analyse des contraintes principales nous permet de déterminer l'état de compression de l'élément. Si tel est le cas, on annule la contrainte principale négative, on obtient ainsi $\left[\sigma_{\text {corrige }}^{*}\right]$. L'analyse des plis (directions, pourcentage) se fait en calculant $[\epsilon]-\left[C^{*}\right]^{-1}\left[\sigma_{\text {corrige }}^{*}\right]$. Ce modèle devient inadapté si les plis deviennent importants (> 
à $5 \%$ ) mais aussi dans le cas des renforts des voiles : en effet, les voiliers renforcent fortement leurs voiles aux points d'ancrage, dont un des objectifs est justement de pouvoir tolérer de la compression.

\subsubsection{Le super-fil}

Dans le modèle membranaire décrit, nous nous sommes efforcés de prendre en compte d'une part, les gréements courant et dormant, et d'autre part, les éléments rigidifiant dans les surfaces souples. L'étai est simulé par des éléments de type superfils : ces éléments sont décrits par deux extrémités (point + quaternion) et un ensemble de points intermédiaires sur une courbe (ordre 2). Une unique tension est calculée pour l'élément super-fil et est traduite en termes de forces en chaque point. Les points intermédiaires simulent donc des poulies idéales sur un câble. Ce modèle est également utilisé pour le glissement le long des poutres comme la liaison guindant-mât, bordurebôme ou latte-voile. Le principal avantage d'utiliser la forme courbe de la poutre, mis à part la meilleure précision de la position des points, réside dans la continuité du glissement d'une poutre à l'autre d'où une meilleure convergence.

\section{Algorithme de couplage dynamique}

Le couplage des deux problèmes exprime les effets de l'interaction. Pour les modèles de structure et de fluide qui ont été choisis, les équations de couplage sont de deux types. Les équations cinématiques imposent la continuité de la vitesse normale des milieux fluide et solide sur l'interface dans sa configuration déformée. Les équations dynamiques décrivent la continuité de l'effort normal sur l'interface : les efforts extérieurs exercés sur la structure sont les efforts aérodynamiques exercés par le fluide parfait sur sa frontière (efforts de pression). Dès lors, le problème complet s'écrit sous la forme d'un ensemble d'équations non linéaires qui peuvent être intégrées dans le temps par une méthode itérative (figure 2). Il est nécessaire de satisfaire exactement l'ensemble des contraintes de compatibilité (continuité géométrique, continuité des vitesses et des contraintes au travers de l'interface) à chaque pas de temps vu la grande flexibilité du milieu solide et la faiblesse du nombre de masse. De plus, il existe des modes de déformation (souple) n'introduisant pas de de variation de l'énergie élastique. Les solutions doivent donc être calculées sur les domaines fluide et solide compatibles de façon totalement implicite (Wuchner et al., 2006). L'intégration implicite des équations sur un pas de temps est en général difficile et coûteuse en raison des non-linéarités intrinsèques des problèmes fluide et solide, et de la non-linéarité géométrique de l'interaction : les équations doivent être résolues sur des domaines dont les géométries sont inconnues. Pour notre structure légère (force d'inertie de la structure largement inférieure aux forces d'inertie du fluide qui l'entoure), le couplage numérique peut diverger très rapidement et il est nécessaire de sous-relaxer la solution $(\zeta=0,1)$ de manière à éviter la divergence du processus ce qui augmente le temps de calcul. La notion de masse ajoutée surfacique calculée à partir du code fluide et introduite dans le code structure permet d'améliorer le conditionnement de la matrice de rigidité globale et stabiliser les calculs pour une convergence rapide en 
s'affranchissant de cette technique de sous-relaxation. Les efforts d'inertie et d'amor-

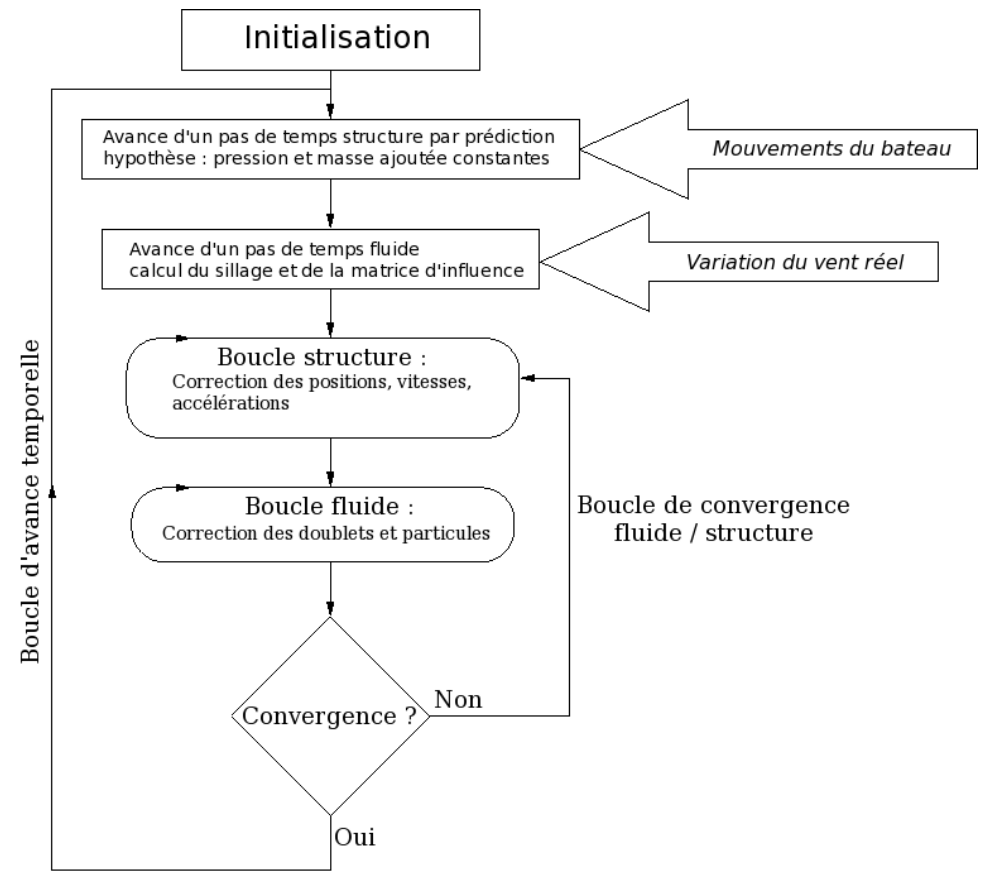

Figure 2. Schéma de couplage

tissement sont également pris en compte dans la résolution dynamique. Une attention toute particulière a été menée sur la correspondance des maillages fluide et solide. Afin d'illustrer la robustesse du schéma, un calcul dynamique a été mené sur le gréement du maxitrimaran Sodebo au cours d'un calcul avec un tangage imposé d'amplitude $A=6^{\circ}$, de période $T=2 \mathrm{~s}$ avec un vent apparent $A W S=20 \mathrm{kt}$ sous une incidence $A W A=28^{\circ}$. Ce cas correspond à un cas extrême en terme de tangage. Une rampe de départ est nécessaire pour initier le mouvement et éviter une accélération trop forte. Après avoir récupéré la $\mathrm{CAO}$ des voiles et les données géométriques et structurelles relatives au gréement, un premier calcul en stationnaire est réalisé. Ce résultat sert à définir les conditions initiales du calcul dynamique. La figure 3 représente le champ de contrainte sur les voiles et le sillage particulaire à trois instants ( $t=0,0 \mathrm{~s}, t=0,7 \mathrm{~s}$ et $t=1,5 \mathrm{~s}$ ) pour ce calcul dynamique. L'intensité des particules discrétisant le sillage est proportionnelle à leurs surfaces. On observe clairement l'évolution du volume des voiles (cambrure, vrillage...) ainsi que les plis. Ces calculs permettent d'accéder d'une part, aux efforts aérodynamiques globaux et d'autre part, aux efforts dans chaque élément des gréements courant et dormant. Ces résultats permettent de mettre en évidence le déphasage entre l'effort propulsif et le mouvement imposé. L'effort augmente brutalement lorsque le mât commence à repartir en arrière c'est-à-dire lorsque l'incidence de l'écoulement apparent augmente. Ce même dépha- 
sage est observé sur la tension d'écoute de grand voile. Cette dernière triple entre les instants $t=0,0 \mathrm{~s}$ et $t=0,7 \mathrm{~s}$ où elle est maximale puis l'écoute devient molle entre les instants $t=1,25 \mathrm{~s}$ et $t=1,7 \mathrm{~s}$ où elle est nulle.
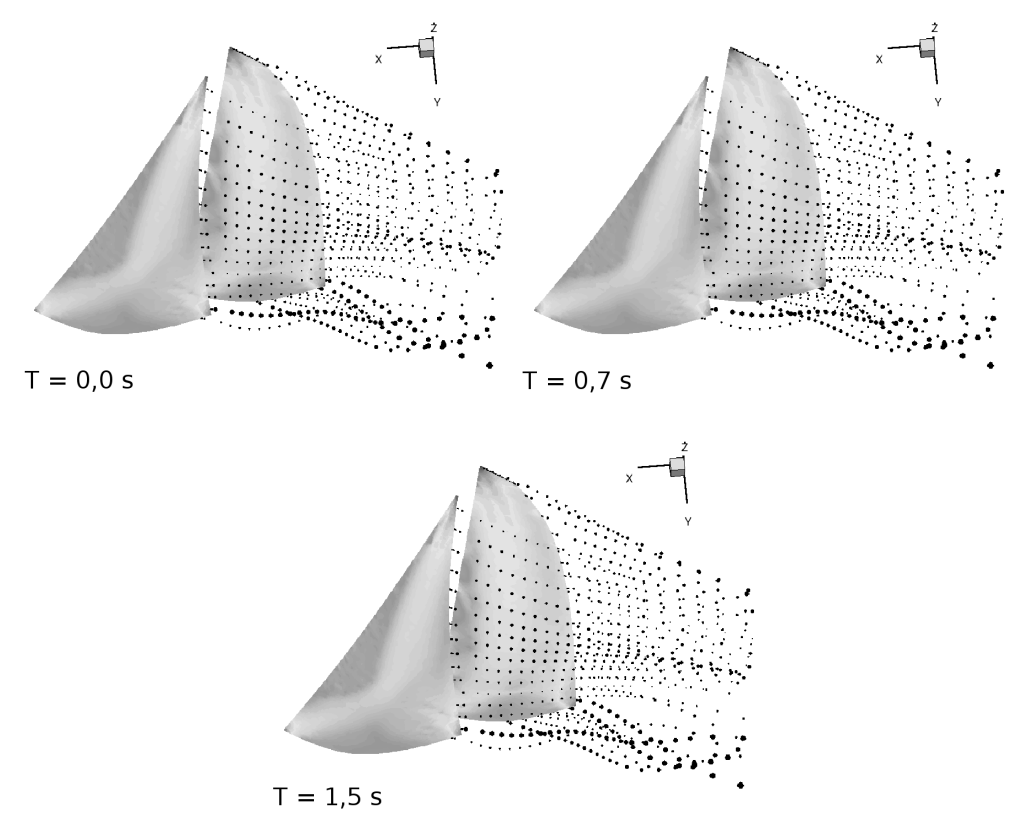

Figure 3. Champ de contrainte sur les voiles et sillage particulaire - $A W S=20 \mathrm{kt}$, $A W A=28^{\circ}$, tangage : $A=6^{\circ}$ et $T=2 \mathrm{~s}$

\section{Mesures expérimentales}

Des études expérimentales sur le comportement mécanique des voiles de bateaux ont déjà donné lieu à plusieurs travaux qui n'ont pas toujours conduit à publication, le secret étant souvent de mise en ce domaine. Citons néanmoins certaines études en soufflerie de l'écoulement autour de voiles bidimensionnelles en nylon imperméable de faible cambrure, avec ou sans mât, dans lesquelles les mesures simultanées globales et/ou locales de pression, vitesse, efforts et une photométrie associée permettent de connaître l'évolution de certains paramètres comme la cambrure maximale et sa position pour différentes incidences et différents Reynolds (Cyr et al., 1996; Weber et al., 2003). Quelques études ont été menées sur un voilier instrumenté en condition réelle de navigation (Masuyama et al., 1997; Claus et al., 2005). L'instrumentation embarquée permettait de connaître en temps réel les efforts dans les gréements courant et dormant, les conditions de navigation (vitesse et position du voilier, vent apparent...) et la forme des voiles par un système de photométrie associé à un posttraitement. Ces mesures sont utilisées comme données d'entrée pour le calcul de l'écoulement, par 
une méthode RANS, autour de ces voiles considérées indéformables dont les formes sont celles en navigation. La difficulté de ce type de calculs réside dans la méconnaissance de l'écoulement autour du gréement, compte tenu des variations, en temps et en espace, de la direction et de la force du vent réel. De plus, les mouvements du navire sous l'effet de la houle et des différentes forces auxquelles est soumis le gréement, peuvent entraîner des mouvements de la tête de mât par rapport au navire. Ils ont des répercussions d'une part, sur la mesure du vent apparent et d'autre part, sur l'évaluation du volume des voiles (photométrie) étant donné que la position (et l'orientation) des caméras nécessaire pour le posttraitement n'est plus connue. Une instrumentation embarquée de ce type est opérationnelle à l'IRENav.

L'objectif à terme des travaux expérimentaux est de pouvoir comparer, pour une configuration donnée, le comportement du gréement prédit par le calcul numérique avec celui qui est observé en réalité. Outre l'instrumentation embarquée dont il est fait référence ci-dessus, une centrale d'attitude a été développée dans le cadre d'un projet mené à l'IRENav sur la dynamique d'une plateforme en mouvement (Durand et al., 2003). Le dispositif expérimental comporte six accéléromètres et trois gyromètres miniatures, de type piézoélectrique, ainsi qu'un système d'acquisition intégré. Un travail important a été réalisé sur le conditionnement et le traitement des signaux afin d'extraire l'information pertinente. En effet, les mouvements du mobile peuvent s'avérer complexes et variés du fait de l'état de la mer et des efforts aérodynamiques et hydrodynamiques qui conduisent à des déplacements significatifs des centres instantanés de rotation. Le principe du traitement des données issues des deux types de capteurs pour fournir une mesure correcte en temps réel des angles de gîte et d'assiette repose sur l'intégration des mesures des gyromètres sur une courte échéance et un ajustement de cette estimation par les données issues des accéléromètres moyennées sur une longue échéance.

Les mouvements du bateau et la variation de vent réel mesurés sont les données d'entrée des codes fluide et structure (voir section 3). Les mouvements du bateau peuvent également être issus d'un calcul hydrodynamique. Dans le but de confronter un résultat de calcul à des mesures en navigation, un essai a été mené sur un J80 équipé de l'instrumentation précédente dans des conditions de vent «musclées » ce qui nous a permis de ne faire que des comparaisons qualitatives sur la forme de la voile et en particulier sur les plis. Dans ces conditions de navigation, le pataras, la bordure, le hale-bas et le cuningham sont pris au maximum et le réglage de la voile se fait principalement sur le chariot d'écoute. Pour la simulation numérique, la voile cross cut est reconstituée à partir de la forme des laizes données par le maître voilier. La matrice de comportement est calculée à partir des tests de traction (chaîne, trame, biais) disponibles. Les sections du mât et de la bôme sont connues (SPARCRAFT) et leurs rigidités ont été calculées puis vérifiées par des mesures lors d'un essai de flexion en deux appuis. Lors de la simulation, le simple fait de tendre le pataras fortement et de reprendre de l'écoute de GV fait apparaître des plis entre le point d'écoute et le milieu du mât. Ces plis, représentés sur la figure 4, sont très visibles d'une part, sur la photo extraite du film de la caméra de tête de mât et d'autre part, sur le résultat de la simulation étant donné que leur taille est largement supérieure à la taille du maillage. 
Lorsque ce n'est pas le cas, comme certains plis le long du mât et de la bôme qui sont observables sur la photo extraite de la vidéo, ils sont calculés et positionnés (zone bleue) à partir d'une analyse des contraintes principales dans chaque élément au cours de la simulation (voir section 2.2).

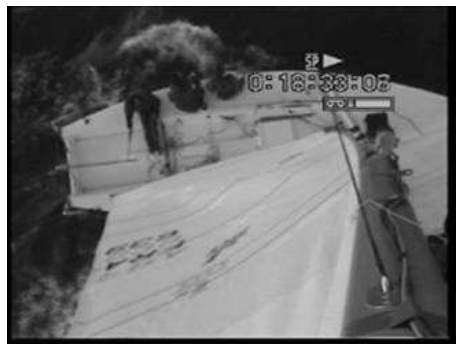

(a)

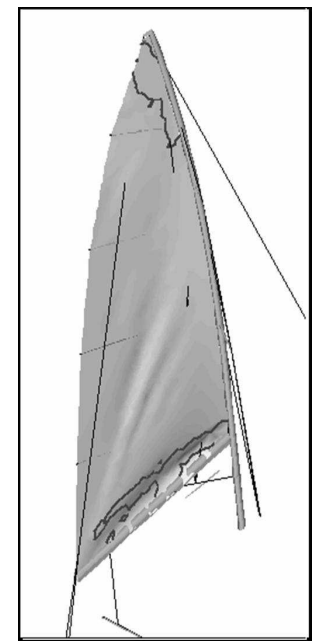

(b)

Figure 4. Comparaison numérique - expérience : J80 en navigation. a) image issue du film pour traitement ISIS. b) résultat de calcul - $A W S=25 \mathrm{kt}, A W A=25^{\circ}$

\section{Conclusion et perspectives}

Cette étude est une contribution à un modèle d'interaction fluide-structure destiné à la prédiction du comportement d'une surface souple de faible épaisseur soumise à un écoulement. Le cadre de validité du modèle est défini par les hypothèses de petites déformations en grands déplacements et de fluide parfait. L'accent a été mis sur le couplage dynamique de l'interaction par un schéma implicite avec la prise en compte d'une masse ajoutée surfacique pour une convergence rapide. Des mesures dans la soufflerie de l'ENSMA de Poitiers et in situ ont été menées dans le but d'accumuler des informations globales et locales dans différentes configurations pour estimer, par une confrontation, la validité des résultats numériques, non plus de manière qualitative mais quantitative. Ces développements restent une base essentielle à la compréhension des divers mécanismes de l'interaction fluide-structure appliquée aux voiles. Ils permettent également, à terme, d'envisager d'optimiser le comportement d'un voilier en navigation réelle et de prendre en compte des variations importantes des caractéristiques des voiles telles que les structures moulées type 3DL ou D4. 


\section{Bibliographie}

Charvet T., Hauville F., Huberson S., "Numerical simulation of the flow over sails in real sailing conditions", Journal of Wind Engineering an Industrial Aerodynamics - Special Issue of Sail Aerodinamics, vol. 63, p. 111-129, 1996.

Claus G., Heisen W., "CFD analysis on the flying shape of modern yacht sails", Actes de The Eleventh International Congress of the International Maritime Association of the Mediterranean, Lisbon, Portugal, September 26-30, 2005.

Cyr S., Newman B. G., "Flow past two-dimensionnal membrane aerofoils with rear separation", Journal of Wind Engineering an Industrial Aerodynamics - Special Issue of Sail Aerodinamics, vol. 63, p. 1-16, 1996.

Durand M., Bot P., Hauville F., Flouriot V., Etude et conception d'un outil d'analyse du comportement dynamique d'une plateforme en mouvement - application aux retournement des multicoques, Rapport de recherche, Contrat d'étude IRENav/SeaSideTech $N^{\circ} \mathrm{W} 0240013$ et $S 0240$ 0273, juin 2003.

Hauville F., Roux Y., « Réglage dynamique d'une voile par une méthode d'interaction fluide structure », Actes des 9e Journées de l'hydrodynamique, Poitiers, France, p. 221-234, 10-12 mars 2003.

Imbert J.-F., Analyse des structures par éléments finis, 3e edn, Cépaduès-Editions, 1995.

Le Maître O., Souza de Cursi J.-E., Huberson S., "Large displacements analysis for ideally flexible sails”, European Journal of Mechanics A/Solids, vol. 17, p. 619-636, 1998.

Masuyama Y., Fukasawa T., "Full scale measurment of sail force and validation of numerrical calculation method", Actes de The Thirteenth Chesepeake Sailing Yacht Symposium, The Society of Naval Architects and Marine Engineers, Annapolis, Maryland, USA, p. 23-36, January 25, 1997.

Mounoury S., Contribution au modèle d'interaction fluide structure de prédiction de comportement d'une surface souple de faible épaisseur soumise à un écoulement; application aux voiles, Thèse de doctorat, Institut de Recherche de l'Ecole Navale, Ecole Centrale de Nantes, 2006.

Mounoury S., Hauville F., Durand M., Astolfi J.-A., Roux Y., « Aéroélasticité non linéaire appliquée aux voiles », Actes des 10e Journées de l'hydrodynamique, Nantes, France, p. 395-408, 7-9 mars 2005.

Rehbach C., Calcul d'écoulements tri-dimensionnels instationnaires de fluide parfait avec nappes tourbillonnaires, Rapport de recherche, Document ONERA - Rapport 10/1737 AY 306, 12/1737 AY 308 et 13/1737 AY 312, 1979 - 1980.

Roux Y., Ba M., Boin J.-P., Guilbaud M., Hauville F., Huberson S., "Sailing boat performance prediction”, Ship Technology Research, vol. 2, n 52, p. 54-64, 2005.

Weber R., Lorillu O., Hureau J., « Elaboration d'un code de calcul d'écoulement autour d'une voile souple $2 \mathrm{D}$ et validation expérimentale », Actes des 9 Journées de l'hydrodynamique, Poitiers, France, p. 207-220, 10-12 mars 2003.

Wuchner R., Kupzok A., Bletzinger K.-U., "Simulation of fluid-structure-interaction with free form membrane structures using an implicit coupling scheme with adaptive under relaxation", Actes de European Conference on Computational Fluid Dynamics-ECCOMAS CFD 2006, Egmond aan Zee, The Netherlands, September 5-8, 2006. 